Original

\title{
Effects of polymerization method on flexural and shear bond strengths of a fiber-reinforced composite resin
}

\author{
Hiroaki Yanagida1), Naomi Tanoue2), Yoshito Minesaki3), Yoko Kamasaki), \\ Taku Fujiwara4), and Hiroyuki Minami1) \\ 1)Department of Fixed Prosthetic Dentistry, \\ Kagoshima University Graduate School of Medical and Dental Sciences, Kagoshima, Japan \\ 2)Division of Pediatric Dentistry, Nagasaki University Hospital, Nagasaki, Japan \\ 3)Fixed Prosthetic Clinic, Kagoshima University Hospital, Kagoshima, Japan \\ 4)Department of Pediatric Dentistry, Nagasaki University Graduate School of Biomedical Sciences, \\ Nagasaki, Japan
}

(Received February 9, 2016; Accepted May 11, 2016)

\begin{abstract}
This study investigated the effects of indirect composite polymerization on the postcuring mechanical properties of a fiber-reinforced composite. An indirect composite seated on glass fibers preimpregnated with polymerized monomer was polymerized by 1) photoirradiation using a halogen-fluorescent polymerizing unit for $5 \mathrm{~min}, 2$ ) method 1 plus secondary heating at $100^{\circ} \mathrm{C}$ for $15 \mathrm{~min}$, 3) photoirradiation using a metal halide light unit for $60 \mathrm{~s}$, or 4) preliminary polymerization using a halogen light unit for $20 \mathrm{~s}$ followed by method 3. After polymerization, the flexural and shear bond strengths of the fiber-reinforced composite were examined, as was the flexural strength of non-fiber-reinforced composite specimens polymerized using the same methods. Among non-fiber-reinforced composite specimens, flexural strength was lower for method 1 than for the other three methods; however, among fiberreinforced composite specimens, the four methods did not significantly differ in flexural strength or shear bond strength. Composite-fiber interface separation
\end{abstract}

Correspondence to Dr. Hiroaki Yanagida, Department of Fixed Prosthetic Dentistry, Kagoshima University Graduate School of Medical and Dental Sciences, 8-35-1 Sakuragaoka, Kagoshima 890-0075, Japan

Fax: +81-99-275-6218

E-mail: yanagida@dent.kagoshima-u.ac.jp

J-STAGE Advance Publication: October 7, 2016

doi.org/10.2334/josnusd.16-0117

DN/JST.JSTAGE/josnusd/16-0117 without breakage of the fiber after flexural strength testing was not observed in specimens polymerized by methods 2 or 4 . In conclusion, use of a conventional unit for high-intensity light irradiation after preliminary irradiation, or light irradiation followed by secondary heating, is recommended for polymerization of composite material seated on polymerized glass fiber.

Keywords: bond strength; glass fiber; indirect composite; polymerization.

\section{Introduction}

Fiber-reinforced composite (FRC) is used as a metal substitute in the production of fixed prostheses. Recent improvements in FRC allow it to be applied not only to crowns but to fixed partial dentures (FPD) (1-4) and interim FPD (5-7), as well. To ensure adequate clinical performance of fixed prostheses made with FRC, the fiber material and resin composite should be bonded and unified both mechanically and chemically.

The three-point bending test is useful in evaluating the clinical performance of FRC-FPD $(2,6,8)$, as it closely simulates occlusal loading on FPD in the oral cavity. A number of factors are thought to be related to the flexural strength of FRC. Obukuro et al. (9) reported that the flexural properties of FRC were significantly associated with the diameter of glass fibers. Tanoue et al. (10) found that composite type affected flexural strength. In addition 
Table 1 Composite materials used

\begin{tabular}{|c|c|c|c|c|}
\hline Trade name & Organic component & Inorganic component & Inorganic content (wt\%) & Manufacturer \\
\hline Estenia $\mathrm{C} \& \mathrm{~B}$ & $\begin{array}{l}\text { UTMA, methacrylate } \\
\text { photopolymerization catalyst }\end{array}$ & $\begin{array}{l}\mathrm{SiO}_{2}, \mathrm{BaO}_{2}, \mathrm{Al}_{2} \mathrm{O}_{3}, \mathrm{La}_{2} \mathrm{O}_{3} \\
\text { (filler size } 2 \mu \mathrm{m}, 0.002 \mu \mathrm{m} \text { ) }\end{array}$ & 92 & $\begin{array}{l}\text { Kuraray Noritake Dental } \\
\text { Inc., Tokyo, Japan }\end{array}$ \\
\hline Estenia C\&B EG Fiber & $\begin{array}{l}\text { UTMA, UDMA, TEGDMA } \\
\text { photopolymerization catalyst }\end{array}$ & long glass fiber, microfiber & 48 & \\
\hline Estenia C\&B Add-on Primer & MTPS, ethanol & & & \\
\hline Estenia C\&B Modeling Liquid & $\begin{array}{l}\text { UTMA, methacylate MDP, } \\
\text { photopolymerization catalyst }\end{array}$ & & & \\
\hline
\end{tabular}

Table 2 Polymerization apparatuses used

\begin{tabular}{llll}
\hline Unit name & Type & Source & Manufacturer \\
\hline$\alpha$-Light IIN & $\begin{array}{l}\text { Photo-polymerization unit } \\
\text { (Box type) }\end{array}$ & $\begin{array}{l}\text { Halogen lamp, 360 W } \times 1 \\
\text { Fluorescent lamp, 27 W } \times 2\end{array}$ & J. Morita Corp., Suita, Japan \\
Hyper LII & $\begin{array}{l}\text { Photo-polymerization unit } \\
\text { (Box type) }\end{array}$ & $\begin{array}{l}\text { Metal halide lamp, } 150 \mathrm{~W} \times 2 \\
\text { (Wavelength: 250-600 nm) }\end{array}$ & Toho Dental Products Co., Ltd., Saitama, Japan \\
Targis Quick & $\begin{array}{l}\text { Photo-polymerization unit } \\
\text { (Open type) }\end{array}$ & $\begin{array}{l}\text { Halogen lamp, 75 W } \times 1 \\
\text { (Wavelength: } 400-500 \mathrm{~nm} \text { ) }\end{array}$ & Ivoclar Vivadent AG, Schaan, Lichtenstein \\
KL 100 & $\begin{array}{l}\text { Heat oven } \\
\text { (Box type) }\end{array}$ & & Kuraray Noritake Dental Inc., Tokyo, Japan \\
\hline
\end{tabular}

to the bending test, shear bond testing is important for direct evaluation of the bond strength between fiber and composite components (11-14).

During the bending and shear bond tests, satisfactory bonding between fiber and composite is necessary in order to ensure adequate mechanical properties of FRC, and many factors that affect this bond have been reported. One study (15) described the effects of composite type on shear bond strength of glass fiber. Another study (16) analyzed the depth at which adhesive resin monomers dissolved into prepolymerized fiber-reinforced composites and reported the efficiency of a secondary interpenetrating polymer network on bonding. Tsushima et al. (17) evaluated the effects of various bonding agents on fiber materials. Clearly, factors required for high bond strengths between fiber and composite have been thoroughly investigated. In general, the mechanical properties of polymerized composite resin materials are related to monomer type (18) and filler type (19).

As mentioned above, resin, fiber, and bonding material components have significant independent effects on FRC properties. However, the polymerization method for each component is also important, as it is related to post-curing properties and the degree of conversion and monomer elution (20). Because FRC prostheses are produced in dental laboratories, the type of laboratory polymerization unit (including the photoirradiation apparatus and heat oven), the combination of units, and the duration of polymerization for each unit are important. Most indirect composite systems use the polymerizing system recommended by the manufacturer. However, laboratory photoirradiation devices vary in the light source used, e.g., halogen bulb, xenon lamp, and fluorescent tube. Each light source has its characteristic wavelength, and optical power and light intensity vary. Although many studies have reported that laboratory polymerization method influences the post-curing properties of indirect composite resin (21-25), few studies have evaluated the effects of polymerization method on the post-curing properties of FRC (15).

This study investigated the effects of polymerization status on the post-curing properties of FRC. The hypotheses tested were 1) that the increased duration of photoirradiation of indirect composite would be associated with FRC flexural strength, 2) that the polymerization method used for the indirect composite would be associated with flexural strength and FRC failure mode, and 3) that the polymerization method used for the indirect composite would be associated with shear bond strength of FRC.

\section{Materials and Methods}

A commercially available indirect composite material (Estenia C\&B, Kuraray Noritake Dental Inc., Tokyo, Japan) and unidirectional monomer-preimpregnated glass fiber material (Estenia C\&B EG Fiber, Kuraray Noritake Dental Inc.) were used for this study. The characteristics of the materials are shown in Table 1.

A 30-mm-long fiber sheaf was placed over a glass plate, and four pieces of $250-\mu \mathrm{m}$-thick gauge were 
placed around the sheaf. This was covered with another glass plate, which was then gently pressed to achieve a unidirectional fiber plate height of $250 \mu \mathrm{m}$. The specimen was then light-polymerized with a light-polymerizing unit (Alpha-Light IIN, J. Morita Corp., Suita, Japan) for $180 \mathrm{~s}$ in accordance with the manufacturer's instructions. The light sources for the light-polymerizing box unit were halogen and fluorescent lamps with a wavelength of 400-600 nm (21-25). The characteristics of this device are shown in Table 2.

The fiber plate was air-abraded (Jet Blast II, J. Morita Corp.) with 50-70- $\mu$ m alumina (Hi-Aluminas, Shofu Inc., Kyoto, Japan) for $10 \mathrm{~s}$. The air pressure was $0.4 \mathrm{MPa}$, and the distance of the nozzle from the fiber surface was approximately $5 \mathrm{~mm}$. After treatment of the surface with an exclusive silane primer (Estenia C\&B Add-on Primer, Kuraray Noritake Dental Inc.), an exclusive bonding agent (Estenia C\&B Modeling Liquid, Kuraray Noritake Dental Inc.) that included 10-methacryloyloxydecyl dihydrogen phosphate as a functional monomer was applied. All processing was performed in accordance with the manufacturer's instructions.

\section{Flexural strength}

The indirect composite material was then placed on the fiber plate and covered with a glass plate to ensure an overall thickness of $2.0 \mathrm{~mm}$, using acrylic spacers. To evaluate the effectiveness of photoirradiation duration, the composite material was polymerized with the aforementioned photopolymerizing unit for 5 min (A5) or 10 min (A10). In addition, to evaluate the polymerization method, the composite material was polymerized with one of the following three polymerization methods: 1) photoirradiation using the photopolymerizing unit for $5 \mathrm{~min}$ and final polymerization using a heat oven (KL 100 , Kuraray Noritake Dental Inc.) at $100^{\circ} \mathrm{C}$ for $15 \mathrm{~min}$ (A5P15; the method recommended by the manufacturer), 2) photoirradiation using a metal halide light unit (Hyper LII, Toho Dental Products Co., Ltd., Saitama, Japan) for $60 \mathrm{~s}(\mathrm{H} 1)$, and 3) preliminary polymerization using a halogen light unit (Targis Quick, Ivoclar Vivadent AG, Schaan, Lichtenstein) for $20 \mathrm{~s}$ and photoirradiation using a metal halide light unit (Hyper LII, Toho Dental Products Co., Ltd.) for $60 \mathrm{~s}$ (TQ0.3H1). The Hyper LII unit is a high-intensity device that has two metal halide lamps with a wavelength of $250-500 \mathrm{~nm}$. The resin materials are exposed in this box unit. The Targis Quick unit is equipped with a halogen lamp with a wavelength of $400-500 \mathrm{~nm}$ and is a preliminary polymerizing unit used to reduce polymerization shrinkage and expose the materials to open air (21-25). These details of these polymerization devices are shown in Table 2.

The fiber-composite plate was mounted in an Isomet low-speed saw (No. 11-1280-170, Buehler Ltd., Evanston, IL, USA) with a diamond wafering blade (No. 11-4244, Buehler Ltd.), perpendicular to the direction of the fiber, and sectioned to a length of $25 \mathrm{~mm}$. The fiber plate was then remounted in the device, horizontal to the fiber direction, and sectioned to obtain rectangular bars measuring $25 \times 2 \times 2 \mathrm{~mm}^{3}$. The prepared specimens were stored in water at $37^{\circ} \mathrm{C}$ for $24 \mathrm{~h}$, and eight specimens were prepared for each polymerization method.

The experimental protocol for evaluation of flexural strength was adapted from ISO 10477:1992(E) for polymer-based crown-and-bridge materials (26). Flexural strength was determined on a universal testing machine (AGS-10kNG, Shimadzu, Kyoto, Japan) at a crosshead speed of $1 \mathrm{~mm} / \mathrm{min}$, using a three-point bending apparatus with two parallel supports at a distance of $20 \mathrm{~mm}$. All the fiber-composite specimens were loaded on the composite material (fiber material on the tension side and composite material on the compression side), and flexural strengths were calculated at the proportional limit. The flexural strength of each method was also measured in non-fiberreinforced specimens, which were used as the control.

For fiber-composite specimens, the tensile stress was loaded until fracture of the composite occurred. Failure mode was determined by observation with an optical microscope (Digital Microscope 400-CAM025, Sanwa Supply, Okayama, Japan) and categorized as (A) composite-fiber interface separation without fiber breakage, (B) composite-fiber interface separation with incomplete fiber breakage, $(\mathrm{C})$ cohesive failure inside the fiber, and (D) complete breakage of composite and fiber. Representative photographs of each failure mode are shown in Fig. 1 (10).

\section{Shear bond strength}

The surface of the polymerized fiber was treated with an exclusive silane primer (Estenia C\&B Add-on Primer) and bonding agent (Estenia C\&B Modeling Liquid) in accordance with the manufacturer's instructions. A piece of $50-\mu \mathrm{m}$-thick tape with a hole (diameter, $5 \mathrm{~mm}$ ) was placed on the surface of the fiber plate to demarcate the bonding area. A brass ring (inside diameter, $6 \mathrm{~mm}$; length, $2 \mathrm{~mm}$; wall thickness, $1 \mathrm{~mm}$ ) was placed around the hole and filled with composite. The resin material was then polymerized under the aforementioned four conditions, except for the method using the exclusive unit for $10 \mathrm{~min}$. In total, 12 specimens were prepared for each polymerization method; however, 36 specimens were prepared for the polymerization method recommended 

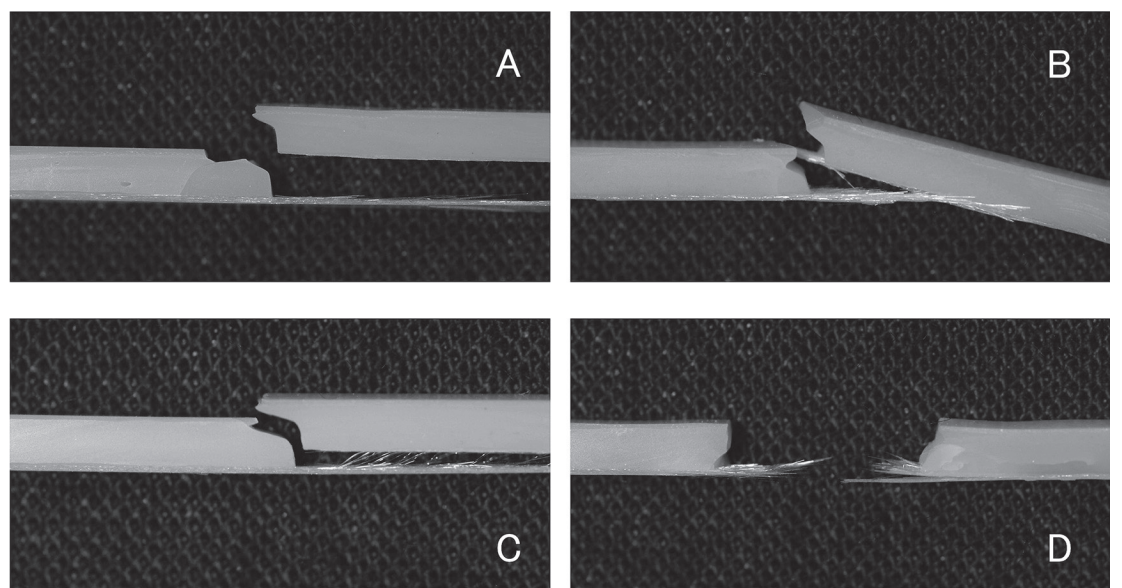

Fig. 1 Representative photographs of failure modes. A: Composite-fiber interface separation without fiber breakage. B: Composite-fiber interface separation with incomplete fiber breakage. C: Cohesive failure inside fiber. D: Complete breakage of composite and fiber. These criteria were developed by Tanoue et al. (10).

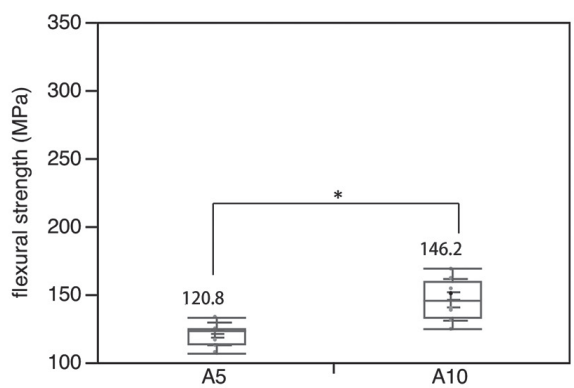

(a)

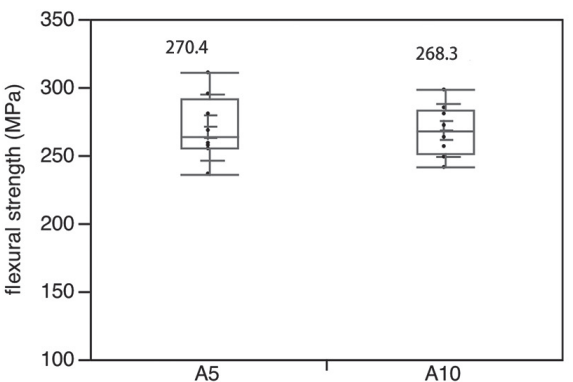

(b)

Fig. 2 Flexural strength after photoirradiation using a halogen-fluorescent polymerizing unit for 5 min (A5) and photoirradiation using the same unit for $10 \mathrm{~min}$ (A10) in non-fiber-reinforced (a) and fiber-reinforced specimens (b). ${ }^{*} P<0.05$.

by the manufacturer (A5P15). After polymerization, the specimens were immersed in water at $37^{\circ} \mathrm{C}$ for $24 \mathrm{~h}$ and embedded in acrylic resin molds.

First, 24 of the 36 A5P15 specimens were assessed, to evaluate the effects of fiber direction on strength. Half (12) the specimens were seated in an ISO/TR 11405 shear-testing jig, to ensure that the fiber direction was perpendicular to the direction of loading. The other half (12) were set in parallel. Shear bond strengths were determined using a mechanical testing device (AGS-10kNG, Shimadzu) at a crosshead speed of $0.5 \mathrm{~mm} / \mathrm{min}$. After confirming that the direction of the fiber was unrelated to strength, the remaining 48 specimens (12 specimens for each of the four polymerization conditions) were tested in the same way, in randomized fiber directions.

To determine failure mode, the fractured surfaces of the specimens (the fiber substructure and composite surfaces) were examined with an optical microscope (Digital Microscope 400-CAM025). In addition, a representative fractured surface was examined using a scanning electron microscope (SEM, S3500N, Hitachi High Technologies Corp., Tokyo, Japan). After shear bond testing, the fractured surfaces were evaluated according to previously described methods $(11,12,14)$.

\section{Statistical analysis}

Mean flexural and shear bond strengths and standard deviations were calculated for each set of specimens. For flexural strength, the Mann-Whitney $U$ test was used to evaluate the two photopolymerization times (A5 and A10), and the Steel-Dwass test was used to evaluate the four polymerizing methods (A5, A5P15, H1, and TQ0.3H1) for each fiber status. For shear bond strength, the effect of fiber direction was analyzed by comparing values using the Mann-Whitney $U$ test, and the effect of polymerization method was analyzed by using the SteelDwass test. Statistical significance was defined as $\alpha=$ 0.05 in all tests. All analyses were done using JMP 10 statistical software (SAS Institute Japan, Tokyo, Japan). 


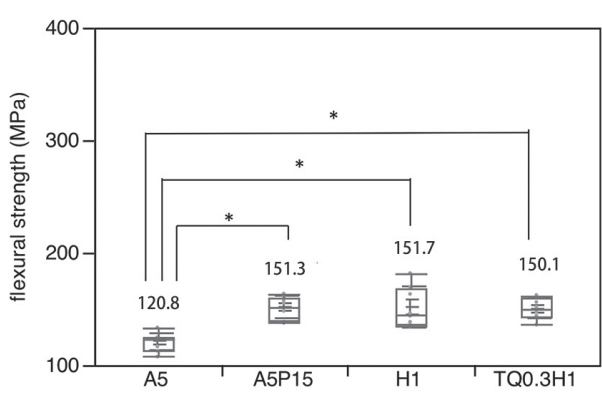

(a)

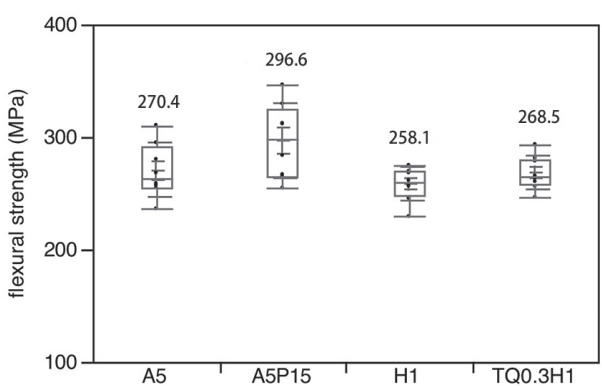

(b)

Fig. 3 Flexural strength after photoirradiation using a halogen-fluorescent polymerizing unit for 5 min (A5), A5 plus secondary heating at $100^{\circ} \mathrm{C}$ for $15 \mathrm{~min}$ (A5P15), photoirradiation using a metal halide light unit for 60 s (H1), and preliminary polymerization using a halogen light unit for $20 \mathrm{~s}$ plus H1 (TQ0.3H1) for non-fiberreinforced (a) and fiber-reinforced specimens (b). $* P<0.05$.

Table 3 Failure mode of fiber-composite specimens

\begin{tabular}{lllll}
\hline \multirow{2}{*}{ Polymerization mode } & \multicolumn{4}{c}{ Category* } \\
\cline { 2 - 5 } & A & B & C & D \\
\hline A5 & 2 & 1 & 5 & 0 \\
A10 & 2 & 4 & 2 & 0 \\
A5P15 & 0 & 2 & 6 & 0 \\
H1 & 3 & 3 & 2 & 0 \\
TQ0.3H1 & 0 & 5 & 3 & 0 \\
\hline
\end{tabular}

*A: composite-fiber interface separation without breakage of the fiber, B: composite-fiber interface separation with incomplete breakage of the fiber, C: cohesive failure inside the fiber, D: complete breakage.

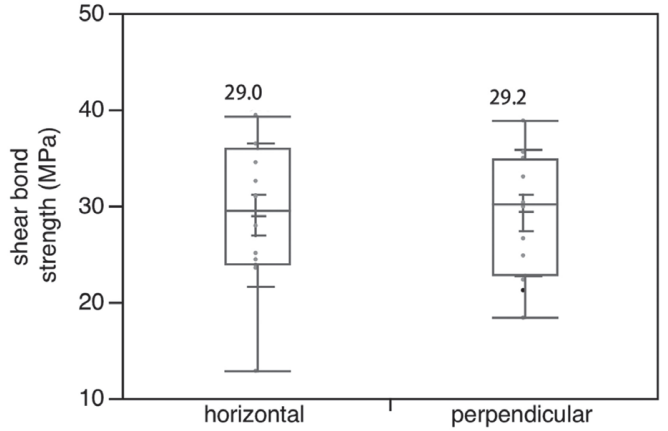

Fig. 4 Shear bond strength for horizontal and perpendicular fiber after photoirradiation using a halogen-fluorescent polymerizing unit for $5 \mathrm{~min}$ and secondary heating at $100^{\circ} \mathrm{C}$ for $15 \mathrm{~min}$. There was no significant difference between groups.

separation without fiber breakage was not seen in A5P15 or TQ0.3H1 specimens.

\section{Shear bond strength}

The results for both fiber directions are shown in Fig. 4. There was no difference between the two shear bond strength values. Furthermore, polymerization method was not associated with shear bond strength (Fig. 5). Optical microscopic observation of failure mode revealed cohesive failure on both sides of the fiber and resin composite in three samples (two in $\mathrm{H} 1$ and one in TQ0.3H1), but cohesive failure of the fiber material was observed in all remaining samples. Images of cohesive failure on both sides of the fiber and resin composite and cohesive failure of the fiber material are shown in Fig. 6.

\section{Discussion}

Flexural strength is one of the most important properties for a $\operatorname{FPD}(2,6,8)$, although many other characteristics are related to clinical performance of a prosthesis. A previous study (10) found that the flexural strength and fracture under all conditions, while composite-fiber interface 


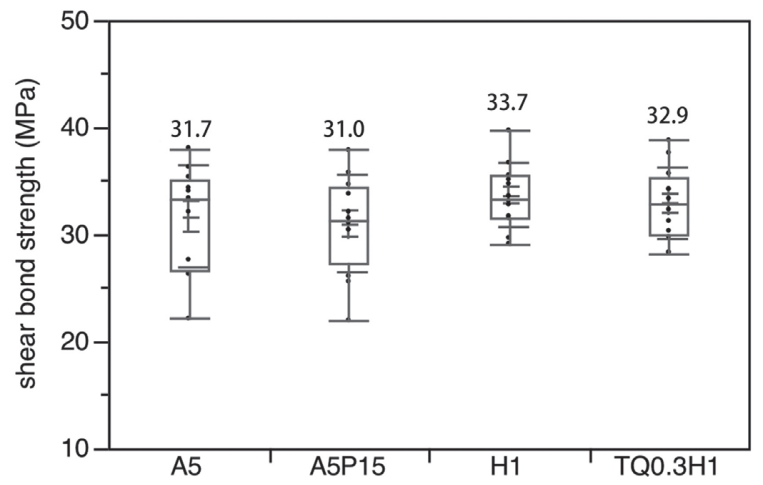

Fig. 5 Shear bond strength after photoirradiation using a halogen-fluorescent polymerizing unit for 5 min (A5), A5 plus secondary heating at $100^{\circ} \mathrm{C}$ for $15 \mathrm{~min}$ (A5P15), photoirradiation using a metal halide light unit for 60 $\mathrm{s}(\mathrm{H} 1)$, and preliminary polymerization using a halogen light unit for $20 \mathrm{~s}$ plus H1 (TQ0.3H1). There was no significant difference between groups.
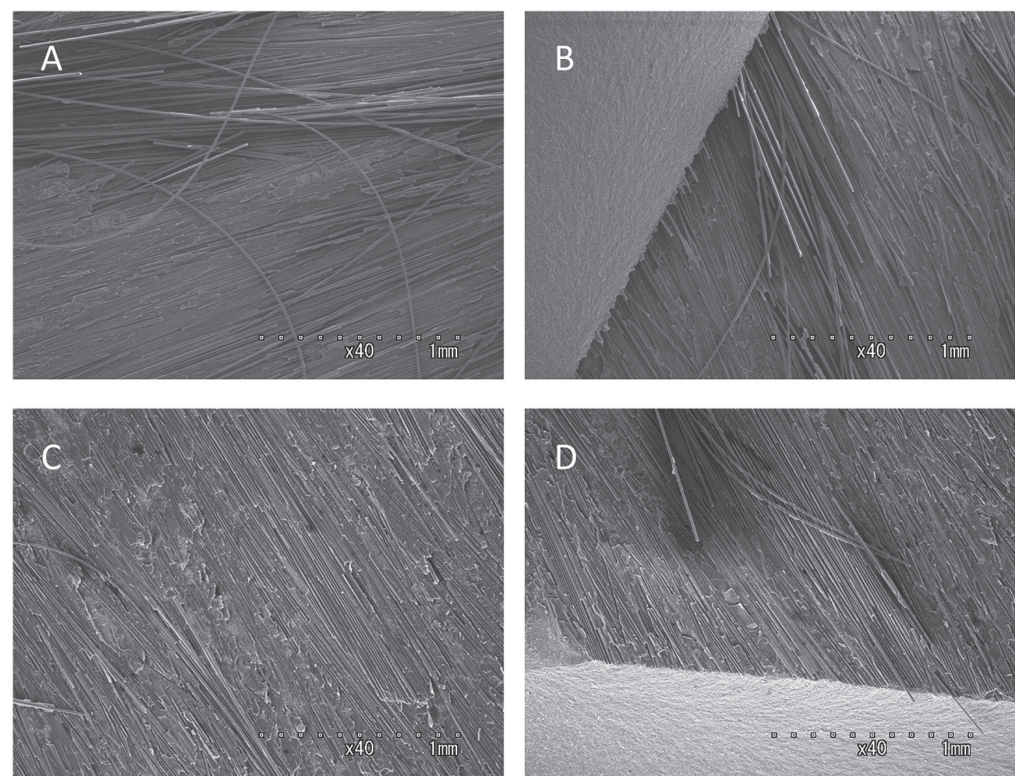

Fig. 6 Scanning electron microscopic images of the composite and fiber surfaces after shear bond testing. A: cohesive failure of the fiber material on the composite side, B: cohesive failure on both sides of the fiber and resin composite on the composite side, $\mathrm{C}$ : cohesive failure of the fiber material on the fiber side, and $\mathrm{D}$ : cohesive failure on both sides of the fiber and resin composite on the fiber side. The fractured composite surface can be seen in B and D. Original magnification $\times 40$.

mode of fiber-composite specimens varied according to composite resin material. In the present study, we examined whether photoirradiation time and polymerization method of composite material were associated with strength. The type of polymerization unit, the combination of units, and polymerization duration using each unit are all important.

Previous studies evaluated the post-curing properties of indirect composite resin materials after three polymerization methods (21-25), and the present fiber-composite specimens were prepared using our previously described methods (10). Finite element analysis revealed that tensile stress concentrations were highest at the bottom of the pontic and that fiber placed from the retainer to the bottom of the pontic increased FPD strength (27). For these reasons, in the present study the fiber material was placed on the tension side and at the bottom of the plate specimen.

The photopolymerizing device used for evaluation of photoirradiation time was equipped with one halogen lamp and two fluorescent tubes as light sources and was used for polymerization of the indirect composite used in this study. Tanoue et al. $(21,23)$ found that the curing depth for several conventional indirect compos- 
ites polymerized with this device was greater than 2 $\mathrm{mm}$ after $90 \mathrm{~s}$ of irradiation. Because the thickness of the composite specimen was $2 \mathrm{~mm}$ in specimens without fiber reinforcement and $1.75 \mathrm{~mm}$ in those with fiber reinforcement, use of this irradiation device for 90 s definitely resulted in penetration to the bottom of specimens. The flexural strength of A5 and A10 FRC specimens, as indicated by strength ratio (10), markedly increased $(223.8 \%$ for A5 and $183.5 \%$ for A10). This finding shows the effectiveness of fiber reinforcement the bonding between the matrix resin of composite and unpolymerized layer of fiber material during irradiation. We believed that an irradiation duration of $5 \mathrm{~min}$, as in this study, would be sufficient for polymerizing the entire resin specimen. However, in tests of indirect resin only (i.e., without fiber reinforcement), flexural strength was significantly increased by extending irradiation time (Fig. 2 ). This indicates that the A5 photopolymerizing method did not result in sufficient conversion.

Without fiber reinforcement, flexural strength was significantly lower for the A5 method than for the other three polymerization methods (Fig. 3). The A5P15 condition was selected for evaluation of secondary heating, $\mathrm{H} 1$ for the effectiveness of a high-intensity light source, and $\mathrm{TQ} 0.3 \mathrm{H} 1$ for evaluation of preliminary polymerization, e.g., soft-start photoactivation (25). Secondary heating after photoirradiation improves post-curing properties (24), and the manufacturer of the indirect composite used therefore recommends the A5P15 method. The disadvantage of secondary heating is increased total polymerization time; the 20-min duration required by the A5P15 status was much longer than that of the other methods. In contrast, a metal halide device equipped with a high-intensity light source can quickly polymerize a photoactivated composite (21) and is effective for establishing adequate post-curing properties (22). However, the $\mathrm{H} 1$ status also has a disadvantage: polymerization shrinkage of the composite (28). The preliminary polymerization of the TQ0.3H1 method was therefore used as a countermeasure against polymerization shrinkage.

The flexural strength values of specimens without fiber reinforcement, using the three methods (i.e., excluding A5), were in the same statistical category. Thus, polymerization of indirect composite might be sufficient with these three methods. The values for TQ0.3H1 and the H1 method were expected to be similar in non-fiber-reinforced cases, because pre-polymerization of TQ0.3H1 was not used for improving mechanical properties but rather for avoiding polymerization shrinkage.

In FRC specimens, flexural strength was not associated with irradiation time or polymerization method
(Figs. 2, 3). In addition, shear bond strength was not associated with polymerization method (Fig. 5). The three study hypotheses regarding FRC specimens were therefore rejected. A previous study (10) found that shear bond strength varied in relation to resin type. When only one composite is used, FRC properties are thought to be affected by the characteristics of the fiber and not by the polymerization method. The superior physical properties of a fiber material outweighed the undesirable composite characteristics caused by insufficient polymerization, even when the fiber was thin (approximately $0.25 \mathrm{~mm}$ ).

Fracture mode varied after tensile stress was loaded until fracture (Table 3), even though all flexural strength values were in the same statistical category. Complete breakage, which is the most undesirable mode, was observed in a composite with low filler content in our previous study but was not observed in this study, probably because of the higher filler content ( $92 \mathrm{wt} \%$ ) in the composite. Nevertheless, composite-fiber interface separation without fiber breakage, which indicates unsatisfactory bonding between the fiber and composite, was observed in A5, A10, and $\mathrm{H} 1$ specimens. In H1 specimens, $37.5 \%$ of specimens exhibited failure mode A, which was the largest proportion among the conditions examined. Interface separation, or adhesive failure, is also unfavorable clinically. Oliveira et al. (28) reported that shrinkage stress increased with higher energy values, while the degree of conversion was not associated with photoactivation mode. Therefore, polymerization shrinkage may adversely affect fiber-composite adhesion. In contrast, no interface separation was observed in TQ0.3H1 specimens, although the light unit used was the same high-intensity unit used for $\mathrm{H} 1$ specimens. The effect of soft-start photoactivation on polymerization shrinkage of composite resin is well known (29), and use of a preliminary polymerizing unit with a lower intensity light source before high-energy irradiation increases shear bond strength between indirect composite and dental metal alloys (25). Combined use of preliminary polymerization using a low-intensity unit and final polymerization using a high-intensity light source is useful in ensuring sufficient bonding between fiber and composite, even though the additional procedure was not directly associated with flexural or shear bond strength.

After shear bond strength testing of the four polymerization conditions, microscopy showed cohesive failure of the fiber material in almost all specimens. This cohesive failure of the fiber material after shear bond strength testing was similar to failure by both interface separation with incomplete fiber breakage and cohesive failure of the fiber component after flexural strength testing of the 
composite-fiber interface but was quite different from failure by composite-fiber interface separation without fiber breakage. The excellent flexural strength of the fiber material could cause composite-fiber interface separation without fiber breakage after the bending test. In any case, we believe that all the present polymerization methods exhibited adequate shear bond strength.

The main limitation of the present study is that only one fiber-reinforced composite system was evaluated. In addition, the duration of water storage was limited. Thus, our findings require confirmation in future studies of different systems with long-term water storage.

Past and present evidence highlights the need to carefully select composite materials with a higher filler content, followed by adequate polymerization, when fiber-reinforced composite is used clinically. For polymerization of the composite material, it is desirable to use high-intensity light irradiation after preliminary irradiation or light irradiation using a conventional unit followed by secondary heating. However, it is more difficult for a highly crosslinked matrix to bond to luting cements and tooth structure; therefore, repair of FRC restorations may be more difficult.

\section{Acknowledgments}

This study was supported in part by Grants-in-Aid for Scientific Research C 15K11168 (2015-2017) and 15K11164 (2015-2017) from the Japan Society for the Promotion of Science (JSPS).

\section{Conflict of interest}

The authors declare that they have no conflict of interest regarding this work.

\section{References}

1. Shinya A, Yokoyama D, Lassila LV, Shinya A, Vallittu PK (2008) Three-dimensional finite element analysis of metal and FRC adhesive fixed dental prostheses. J Adhes Dent 10, 365-371.

2. van Dalen A, Feilzer AJ, Kleverlaan CJ (2008) In vitro evaluation of failure loads of nonmetal cantilevered resin-bonded fixed dental prostheses. J Adhes Dent 10, 461-469.

3. Nejatidanesh F, Momeni G, Savabi O (2009) Flexural strength of interim resin materials for fixed prosthodontics. J Prosthodont 18, 507-511.

4. Keulemans F, Van Dalen A, Kleverlaan CJ, Feilzer AJ (2010) Static and dynamic failure load of fiber-reinforced composite and particulate filler composite cantilever resin-bonded fixed dental prostheses. J Adhes Dent 12, 207-214.

5. Balkenhol M, Ferger P, Mautner MC, Wöstmann B (2007) Provisional crown and fixed partial denture materials: mechanical properties and degree of conversion. Dent Mater 23, 1574-1583.

6. Garoushi SK, Vallittu PK, Lassila LV (2008) Short glass fiber-reinforced composite with a semi-interpenetrating polymer network matrix for temporary crowns and bridges. J Contemp Dent Pract 9, 14-21.

7. Jo LJ, Shenoy KK, Shetty S (2011) Flexural strength and hardness of resins for interim fixed partial dentures. Indian J Dent Res 22, 71-76.

8. Costa AK, da Silva LH, Saavedra GS, Paes TJ Jr, Borges AL (2012) Flexural strength of four adhesive fixed dental prostheses of composite resin reinforced with glass fiber. J Adhes Dent 14, 47-50.

9. Obukuro M, Takahashi Y, Shimizu H (2008) Effect of diameter of glass fibers on flexural properties of fiber-reinforced composites. Dent Mater J 27, 541-548.

10. Tanoue N, Sawase T, Matsumura H, McCabe JF (2012) Properties of indirect composites reinforced with monomerimpregnated glass fiber. Odontology 100, 192-198.

11. Behr M, Rosentritt M, Latzel D, Kreisler T (2001) Comparison of three types of fiber-reinforced composite molar crowns on their fracture resistance and marginal adaptation. J Dent 29, 187-196.

12. Lastumäki TM, Lassila LV, Vallittu PK (2003) The semiinterpenetrating polymer network matrix of fiber-reinforced composite and its effect on the surface adhesive properties. $\mathrm{J}$ Mater Sci Mater Med 14, 803-809.

13. Lassila LV, Tezvergil A, Dyer SR, Vallittu PK (2007) The bond strength of particulate-filler composite to differently oriented fiber-reinforced composite substrate. J Prosthodont $16,10-17$.

14. Antonopoulou A, Papadopoulos T, Hatzikyriakos A (2012) In vitro evaluation of shear bond strength and mode of failure of the interface between an indirect composite bonded to fiber-reinforced composite substructures. J Prosthodont 21, 451-459.

15. Polacek P, Pavelka V, Ozcan M (2013) Adhesion of resin materials to S2-glass unidirectional and E-glass multidirectional fiber reinforced composites: effect of polymerization sequence protocols. J Adhes Dent 15, 507-510.

16. Wolff D, Geiger S, Ding P, Staehle HJ, Frese C (2012) Analysis of the interdiffusion of resin monomers into prepolymerized fiber-reinforced composites. Dent Mater 28, 541-547.

17. Tsushima S, Gomi H, Shinya A, Yokoyama D, Vallittu PK, Shinya A (2008) Effect of commercially available bonding agents impregnated with fibers on bending strength of hybrid resin. Dent Mater J 27, 723-729.

18. Gonçalves F, Kawano Y, Pfeifer C, Stansbury JW, Braga RR (2009) Influence of BisGMA, TEGDMA, and BisEMA contents on viscosity, conversion, and flexural strength of experimental resins and composites. Eur J Oral Sci 117, 442-446.

19. Garoushi S, Lassila LV, Vallittu PK (2011) Influence of nanometer scale particulate fillers on some properties of microfilled composite resin. J Mater Sci Mater Med 22, 16451651.

20. Randolph LD, Palin WM, Bebelman S, Devaux J, Gallez B, 
Leloup G et al. (2014) Ultra-fast light-curing resin composite with increased conversion and reduced monomer elution. Dent Mater 30, 594-604.

21. Tanoue N, Matsumura H, Atsuta M (1998) Curing depth of a composite veneering material polymerized with seven different laboratory photo-curing units. J Oral Rehabil 25, 199-203.

22. Tanoue N, Matsumura H, Atsuta M (1998) Properties of four composite veneering materials polymerized with different laboratory photo-curing units. J Oral Rehabil 25, 358-364.

23. Tanoue N, Matsumura H, Atsuta M (1999) Curing depth of prosthetic composite materials polymerized with their proprietary photo-curing units. J Oral Rehabil 26, 594-599.

24. Tanoue N, Matsumura H, Atsuta M (2000) Comparative evaluation of secondary heat treatment and a high intensity light source for the improvement of properties of prosthetic composites. J Oral Rehabil 27, 288-293.

25. Tanoue N, Shimoe S, Atsuta M, Matsumura H (2005)
Application of a preliminary light-curing unit for enhanced bonding between a gold alloy and veneering materials. Am J Dent 18, 82-86.

26. International Organization for Standardization (1992) Dentistry--Polymer-based crown and bridge materials. ISO 10477:1992, Geneve.

27. Nakamura T, Ohyama T, Waki T, Kinuta S, Wakabayashi K, Takano N et al. (2005) Finite element analysis fiber-reinforced fixed partial dentures. Dent Mater J 24, 275-279.

28. Oliveira KM, Lancellotti AC, Ccahuana-Vásquez RA, Consani S (2012) Shrinkage stress and degree of conversion of a dental composite submitted to different photoactivation protocols. Acta Odontol Latinoam 25, 115-122.

29. Alonso RC, Correr GM, Cunha LG, De Moraes Souto Pantoja CA, Puppin-Rontani RM, Sinhoreti MA (2007) Modulated photoactivation methods--effect on marginal and internal gap formation of restorations using different restorative composites. J Biomed Mater Res B Appl Biomater 82, 346-351. 